\title{
Observational cohort study focused on treatment continuity of patients administered XELOX plus bevacizumab for previously untreated metastatic colorectal cancer
}

\author{
This article was published in the following Dove Press journal: \\ OncoTargets and Therapy \\ 7 July 2016 \\ Number of times this article has been viewed
}

\author{
Masahito Kotaka' \\ Fusao Ikeda ${ }^{2}$ \\ Masaki Tsujie ${ }^{3}$ \\ Shinichi Yoshioka ${ }^{4}$ \\ Yoshihiko Nakamoto ${ }^{5}$ \\ Takaaki Ishii ${ }^{6}$ \\ Takahisa Kyogoku ${ }^{7}$ \\ Takeshi Kato ${ }^{8}$ \\ Akihito Tsuji ${ }^{9}$ \\ Michiya Kobayashi ${ }^{10}$ \\ 'Gastrointestinal Cancer Center, Sano \\ Hospital, Kobe, Hyogo, ${ }^{2}$ Department \\ of Surgery, Kohka Public Hospital, \\ Koka, Shiga, ${ }^{3}$ Department of Colorectal \\ Surgery, Sakai City Medical Center, \\ Sakai, Osaka, ${ }^{4}$ Department of Surgery, \\ Hyogo Prefectural Nishinomiya \\ Hospital, Nishinomiya, Hyogo, \\ ${ }^{5}$ Department of Surgery, Seikei-kai \\ Chiba Medical Center, Chiba, Chiba, \\ ${ }^{6}$ Department of Surgery, Kobe-Ekisaikai \\ Hospital, Kobe, Hyogo, ${ }^{7}$ Department \\ of Surgery, Nishikobe Medical \\ Center, Kobe, Hyogo, ${ }^{8}$ Department \\ of Colorectal Surgery, Kansai Rosai \\ Hospital, Amagasaki, Hyogo, ${ }^{9}$ Kagawa \\ University Hospital Cancer Center, \\ Kita-gun, Kagawa, ${ }^{10}$ Department of \\ Human Health and Medical Sciences, \\ Kochi Medical School, Kochi University, \\ Nangoku, Kochi, Japan
}

Correspondence: Masahito Kotaka Gastrointestinal Cancer Center, Sano Hospital, 2-5-I Shimizugaoka, Tarumi-ku, Kobe, Hyogo 655-003I, Japan

$\mathrm{Tel}+8 \mathrm{I} 787851000$

Fax +8I 787850077

Email tomomakotaka64I0@yahoo.co.jp
Background: There has been remarkable progress in systemic chemotherapy for metastatic colorectal cancer due to the widespread use of irinotecan, oxaliplatin, anti-vascular endothelial growth factor antibody, and anti-epidermal growth factor receptor antibody. It is important to continue treatment with the optimal combination of these drugs and prolong progression-free survival (PFS) to improve overall survival (OS). We conducted a prospective observational cohort study of 40 patients treated with XELOX plus bevacizumab for previously untreated metastatic colorectal cancer to investigate treatment continuity.

Patients and methods: Eligibility criteria were as follows: 1) histologically confirmed metastatic colorectal cancer; 2) lesions evaluable by imaging; 3) previously untreated; 4) suitable condition to receive XELOX plus bevacizumab; and 5) written informed consent. Outcomes were treatment continuity, overall response rate, resection rate, liver resection rate, time to treatment failure, PFS, and OS. Forty patients were enrolled and followed up for 2 years.

Results: Between July 2010 and June 2012, 40 patients were enrolled. The median number of treatment cycles was 7.5, and the reasons for discontinuation of treatment were as follows: complete response (five patients), resection (ten patients), progression (15 patients), adverse events (seven patients), and patient refusal (three patients). The overall response rate was $57.5 \%$, resection rate was $25 \%$, and liver resection rate was $15 \%$. After a median follow-up of 31.4 months, the median time to treatment failure, PFS, and OS were 5.3, 13.3, and 38.9 months, respectively.

Conclusion: Although the median time to treatment failure was 5.3 months, the median PFS and OS were prolonged to 13.3 and 38.9 months, respectively. This may have resulted from the chemotherapy-free interval due to complete response in five patients and resection in ten patients.

Keywords: observational cohort study, metastatic colorectal cancer, XELOX plus bevacizumab, treatment continuity

\section{Introduction}

There has been remarkable progress in systemic chemotherapy for metastatic colorectal cancer (mCRC) due to the widespread use of irinotecan, oxaliplatin, anti-vascular endothelial growth factor antibody, and anti-epidermal growth factor receptor antibody. ${ }^{1-6}$ Recently, there have been additional advances with the advent of new drugs, such as regorafenib and TAS-102. ${ }^{7,8}$ Several randomized clinical studies have been performed to identify the optimal combination of these drugs for use as first-line treatment. ${ }^{9-11}$

It is important to continue first-line treatment and prolong progression-free survival (PFS) to improve the rate of overall survival (OS) as a true endpoint in the treatment of $\mathrm{mCRC}$. 
PFS is a surrogate endpoint for OS for mCRC. ${ }^{12,13}$ The French clinical study group GERCOR conducted the OPTIMOX 1 study to assess planned discontinuation and reintroduction of oxaliplatin in a stop-and-go strategy, and the OPTIMOX 2 study to evaluate the role of chemotherapy-free intervals (CFI), known as "chemotherapy holidays". ${ }^{14,15}$ First-line treatment strategy with maintenance therapy has also been evaluated. In the CAIRO 3 study and the AIO KRK 0207 study, maintenance therapy with fluoropyrimidine-based chemotherapy plus bevacizumab and observation were compared in patients with mCRC without disease progression after a certain number of cycles of oxaliplatin-based chemotherapy with bevacizumab. ${ }^{16,17}$

It is important to consider the treatment strategy to maintain the duration of first-line treatment, which leads to improved PFS and OS in patients with mCRC. We conducted a prospective observational cohort study of 40 patients treated with XELOX plus bevacizumab for previously untreated $\mathrm{mCRC}$ to investigate treatment continuity in daily clinical practice.

\section{Patients and methods}

This was a prospective observational cohort study to investigate the data from consecutive patients treated with XELOX plus bevacizumab during a certain period in several institutions in Japan.

This study was conducted in compliance with the Declaration of Helsinki and the Ethical Guidelines on Clinical Studies after receiving approval from the ethics committees of all participating institutions (Sano Hospital, Kobe City Medical Center West Hospital, Kobe-Ekisaikai Hospital, Nishikobe Medical Center, and Hyogo Prefectural Nishinomiya Hospital). Written informed consent was obtained from all patients before enrollment. The study registration was as follows: University Hospital Medical Information Network Clinical Trials Registry (UMIN000003416).

\section{Patient selection}

The inclusion criteria were as follows regardless of sex and age:

1. mCRC confirmed cytologically and/or histologically

2. lesions evaluable by imaging

3. previously untreated (except for surgery)

4. suitable condition to receive XELOX plus bevacizumab

5. written informed consent.

The exclusion criteria were as follows:

1. other active malignancies (ie, diagnosed within 5 years)

2. contraindications for bevacizumab, oxaliplatin, and capecitabine

3. considered unsuitable for the study by the investigators.

\section{Treatment}

Each patient received XELOX plus bevacizumab. On day 1, bevacizumab $(7.5 \mathrm{mg} / \mathrm{kg})$ and oxaliplatin $\left(130 \mathrm{mg} / \mathrm{m}^{2}\right)$ were injected intravenously. From day 1 to day 14, capecitabine $\left(2,000 \mathrm{mg} / \mathrm{m}^{2} /\right.$ day $)$ was orally administered. Each cycle was repeated every 3 weeks.

Laboratory test including serological tumor marker was repeated for every cycle, and adverse event grades were evaluated in accordance with the National Cancer Institute Common Terminology Criteria for Adverse Events, version 3.0.

Tumor assessment was repeated every $8( \pm 2)$ weeks using the same radiological imaging as baseline, basically using the contrast enhanced computed tomography (chest, abdominal, and pelvic), and assessed by the investigator based on the Response Evaluation Criteria in Solid Tumors, version 1.0 .

\section{Statistical considerations}

The outcomes were treatment continuity, overall response rate, resection rate, liver resection rate, time to treatment failure (TTF), PFS, and OS.

Forty patients were enrolled and followed up for 2 years.

TTF was defined as the time from treatment initiation to disease progression or death from any cause or treatment discontinuation before completion, whichever was earlier. PFS was defined as the time from treatment initiation to disease progression or death from any cause, whichever was earlier. OS was defined as the time from treatment initiation to death from any cause.

TTF, PFS, and OS were estimated using the KaplanMeier method.

\section{Results}

Between July 2010 and June 2012, 40 patients were registered in this study from five institutions in Japan.

\section{Patient characteristics}

Of the 40 patients, 24 were male, and their median age was 63 years (range 35-84 years). The numbers of patients with the ECOG Performance Status 0, 1, and 2 were 33, 6, and 1, respectively; 24 patients had colon cancer; the primary tumor was resected in 35 patients before the commencement of XELOX plus bevacizumab; 23 patients had liver metastases; 16 had lung metastases; four had lymph node metastases; seven had peritoneal metastases; seven had other site metastases; 14 had wild-type $K R A S$ Exon 2 tumor; 15 had mutant $K R A S$ Exon 2 tumor; and eleven were untested. 


\section{Treatment continuity}

Figure 1 shows treatment continuities of XELOX plus bevacizumab in the 40 patients. The median number of treatment cycles was 7.5 (range 2-40). The reasons for treatment discontinuation were as follows: complete response (CR), five patients $(12.5 \%)$; resection, ten patients $(25 \%)$; disease progression, 15 patients (37.5\%); adverse events, seven patients (17.5\%); and patient refusal, three patients (7.5\%).

Among the seven patients who discontinued treatment due to adverse events, two discontinued due to grade 2 peripheral neuropathy, two due to grade 2 allergy, one due to both grade 3 hypertension and grade 2 allergy, one due to grade 3 nausea and anorexia, and one due to grade 2 hand-foot syndrome, respectively. Otherwise, no noteworthy adverse events happened and were reported to continue treatment with XELOX plus bevacizumab.

Figure 2 shows the details of a patient who discontinued treatment because of radiological CR. He was a 35-year-old man with synchronous liver metastases from colon cancer, who started treatment with XELOX plus bevacizumab after colorectomy, and achieved a partial response after two cycles and CR after 17 cycles. He had a PFS of 39 months after the initiation of XELOX plus bevacizumab.
Figure 3 shows the details of a patient who continued treatment until resection. She was a 60 -year-old woman with metachronous paraaortic lymph node metastases from rectal cancer, who achieved a partial response after four cycles of XELOX plus bevacizumab, underwent resection of paraaortic lymph node metastases, and achieved pathological CR. She had a disease-free survival of 30 months after XELOX plus bevacizumab initiation.

\section{Treatment outcome}

The overall response rate was $57.5 \%(\mathrm{CR}$, seven patients [17.5\%]; partial response, 16 patients [40.0\%]). Ten patients had stable disease (25.0\%), five had progression disease (PD) (12.5\%), and two were not evaluated (5.0\%).

The resection rate was $25.0 \%$ (liver metastases in six patients, lung metastases in two patients, paraaortic lymph node metastases in one patient, and local recurrence in one patient) and the liver resection rate was $15.0 \%$ (six patients).

After a median follow-up period of 31.4 months (range 0.7-50.9 months), the median TTF, PFS, and OS were, respectively, 5.3 months $(95 \%$ confidence interval [CI], 3.6-9.1 months) (Figure 4), 13.3 months (95\% CI,

Number of XELOX + BV treatment cycles

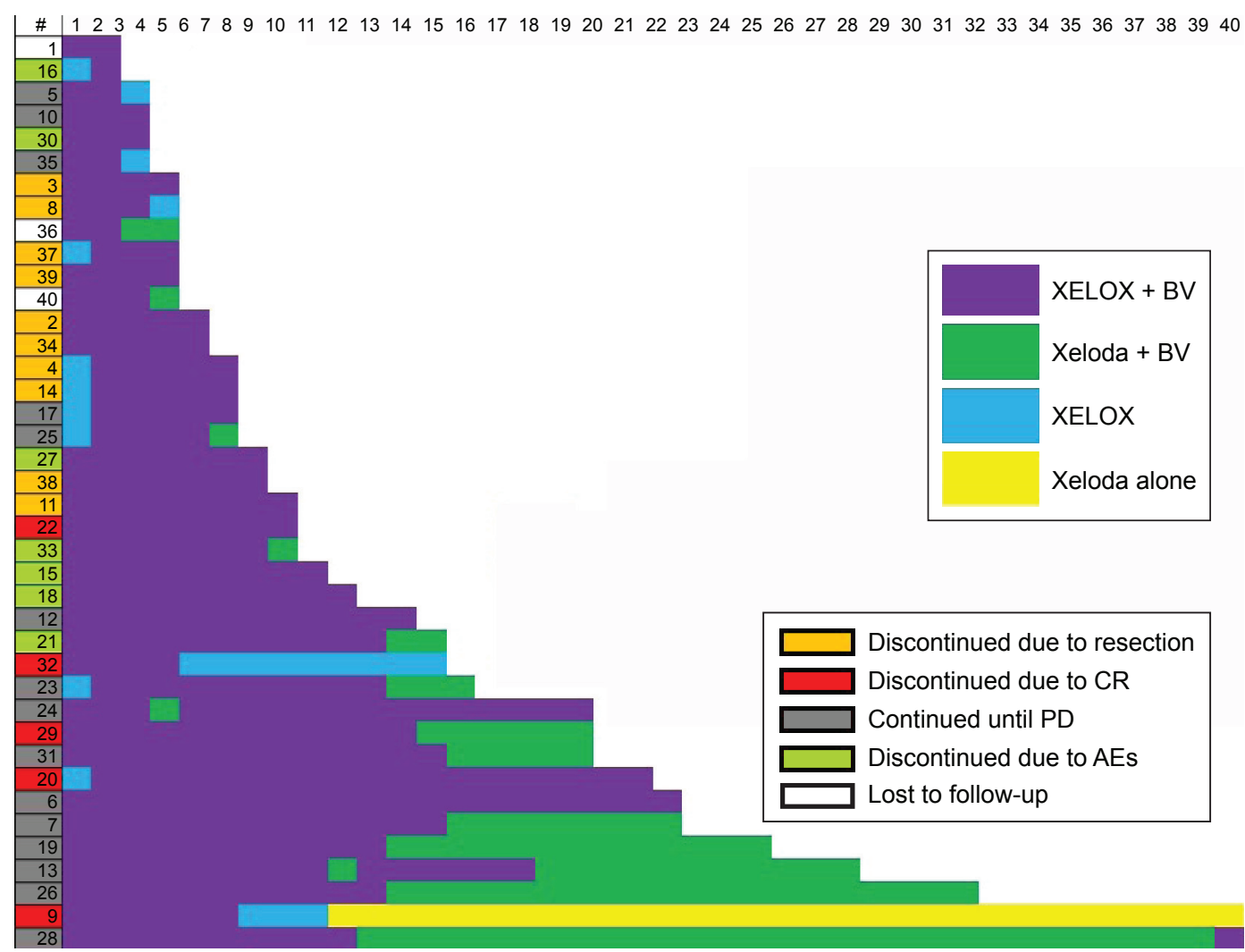

Figure I Treatment continuity $(n=40)$.

Note: 40 patients received a median number of 7.5 cycles of XELOX $+B V ;$ range $=2-40$ cycles.

Abbreviations: BV, bevacizumab; CR, complete response; PD, progression disease; AEs, adverse events. 


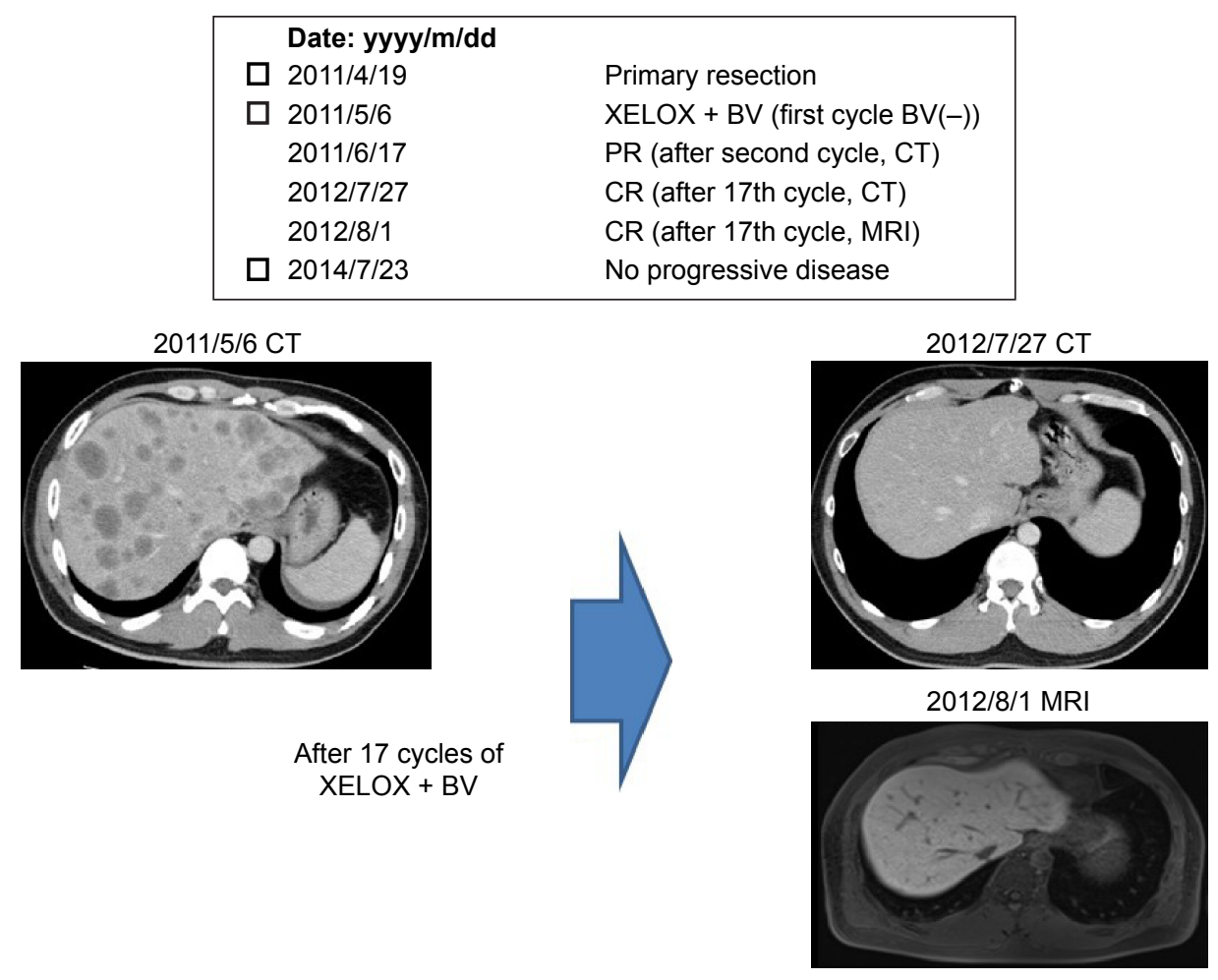

Figure 2 Case I (\#20): liver metastases from colon cancer in a 35-year-old man.

Abbreviations: BV, bevacizumab; PR, partial response; CT, computed tomography; CR, complete response; MRI, magnetic resonance imaging.

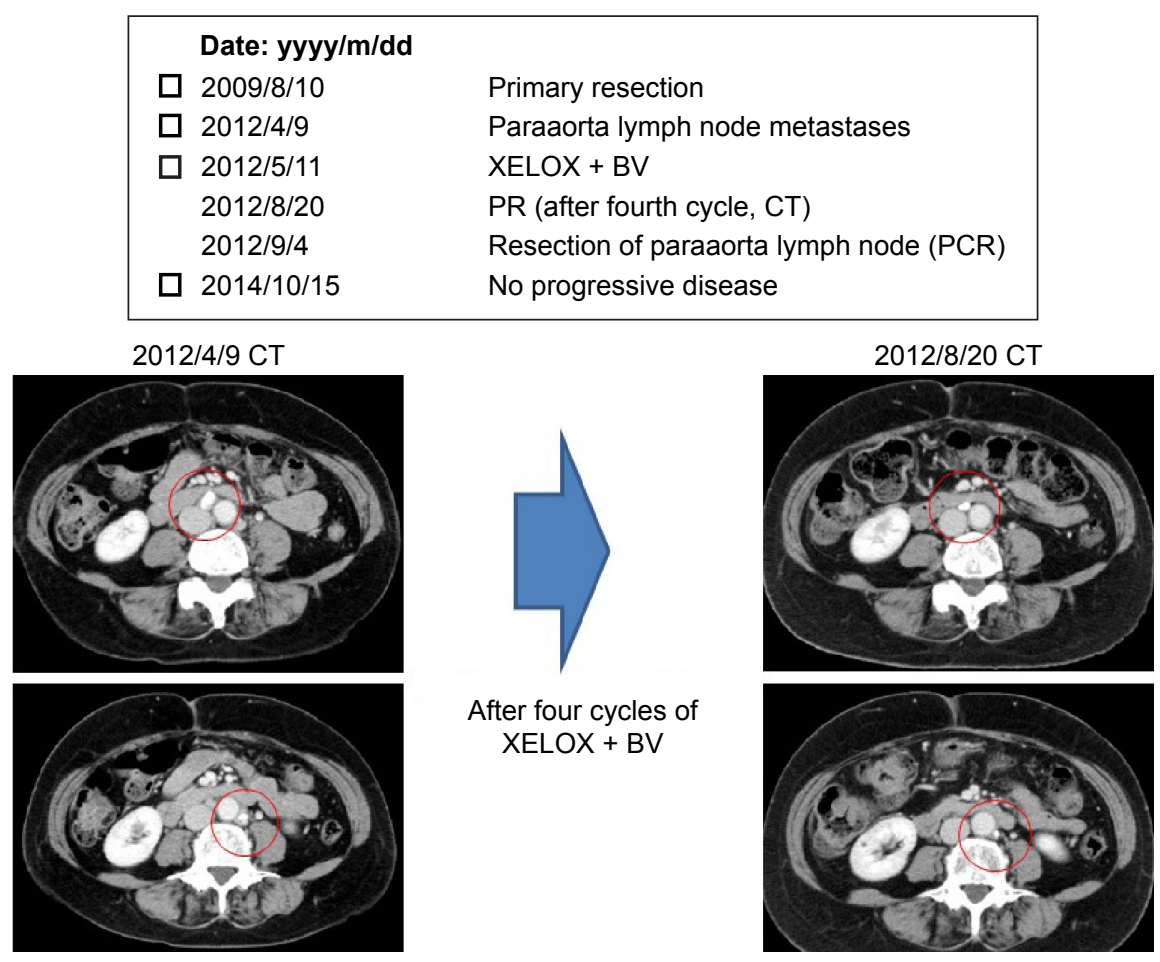

Figure 3 Case 2 (\#39): paraaortic lymph node metastases from rectal cancer in a 60-year-old woman.

Note: Red circles indicate metastases.

Abbreviations: BV, bevacizumab; PR, partial response; CT, computed tomography; PCR, polymerase chain reaction. 


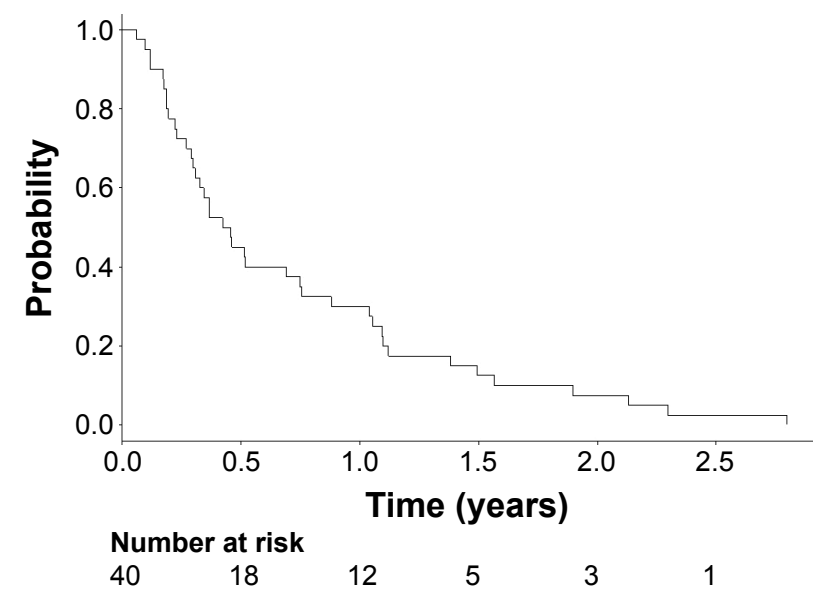

Figure 4 Time to treatment failure (5.3 months [range 3.6-9.1 months]).

9.8-18.2 months) (Figure 5), and 38.9 months (95\% CI, 23.5 months to not reached) (Figure 6).

\section{Discussion}

This study was performed to evaluate treatment continuity for $\mathrm{mCRC}$ patients with introduction of maintenance therapy in daily clinical practice.

The overall treatment outcome of XELOX plus bevacizumab is almost similar to that of the previous studies. The overall response rate, $57.5 \%$, is consistent compared to NO16966 (47\%; XELOX \pm bevacizumab) and JO19380 (72\%; XELOX + bevacizumab). ${ }^{18,19}$ The median PFS and OS (13.3 and 38.9 months, respectively) tend to be slightly improved compared to NO16966 (8.0 and 19.8 months; XELOX \pm bevacizumab) and JO19380 (11.0 and 27.4 months; XELOX + bevacizumab). ${ }^{18,19}$

The median number of treatment cycles was 7.5 , and only 14 patients $(35 \%)$ were treated with capecitabine for maintenance therapy with or without bevacizumab followed by induction of XELOX plus bevacizumab. Among 20 patients

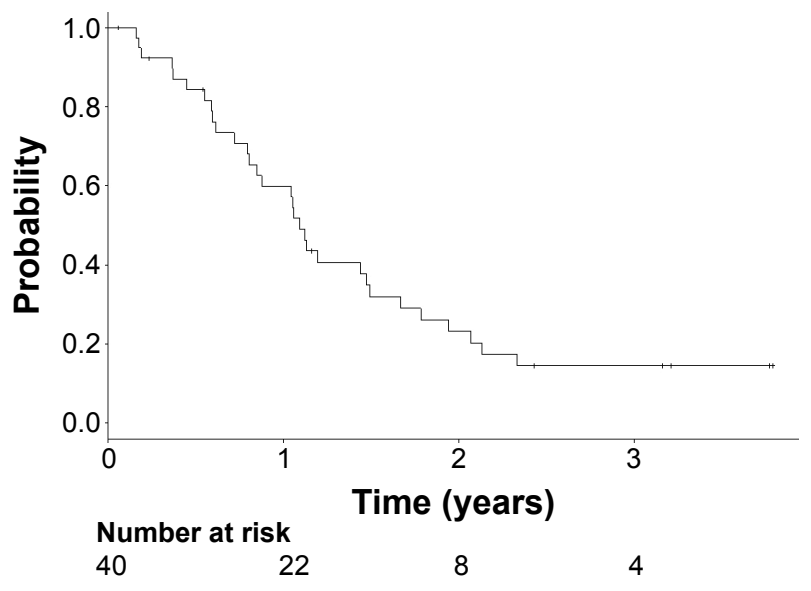

Figure 5 Progression-free survival (|3.3 months [range 9.8-18.2 months]).

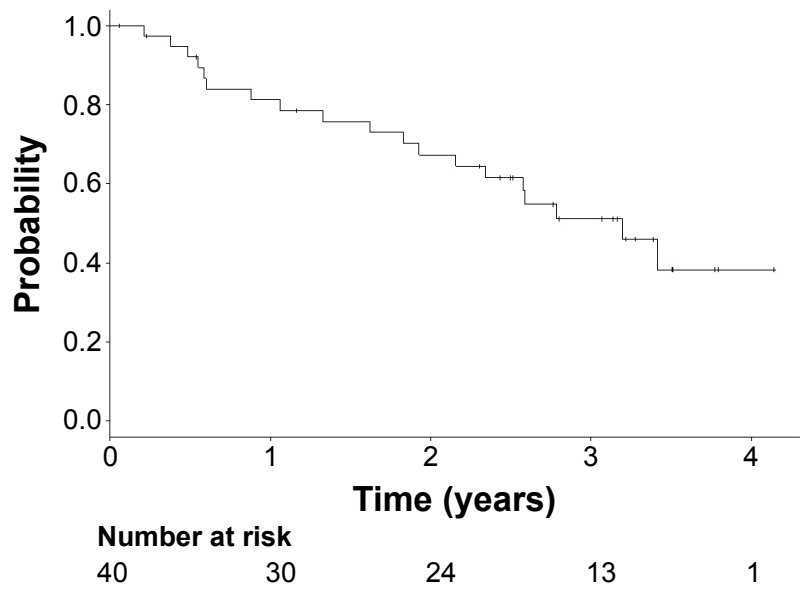

Figure 6 Overall survival (38.9 months [range 23.5 to NR months]). Abbreviation: NR, not reached.

who received eight or more cycles of XELOX plus bevacizumab, eleven patients (55\%) received maintenance therapy with capecitabine with or without bevacizumab. Among 20 patients who received seven or fewer cycles, secondary resection was performed in nine patients (45\%) (Figure 1).

It is important to continue first-line treatment and prolong PFS to improve OS in patients with mCRC. Therefore, the following two treatment strategies may be useful: 1) discontinuation of a cytotoxic agent and switch from induction to maintenance therapy, and 2) a chemotherapy holiday.

The French clinical study group GERCOR conducted the OPTIMOX 1 study to assess planned discontinuation and reintroduction of oxaliplatin in a stop-and-go strategy, and the OPTIMOX 2 study to evaluate the role of a chemotherapy holiday. ${ }^{14,15}$ The OPTIMOX 1 study compared FOLFOX4 until progression with FOLFOX7 for six cycles, followed by maintenance therapy with 5-FU/LV alone and reintroduction of FOLFOX7. Median duration of disease control, a primary endpoint in this study, was 9.0 months in patients treated with continuous FOLFOX4, compared with 10.4 months in patients treated with intermittent FOLFOX7 in a stop-and-go strategy $(P=0.89)$. This study showed no difference in efficacy between continuous chemotherapy and intermittent chemotherapy with maintenance therapy. ${ }^{14}$ On the other hand, the OPTIMOX 2 study compared mFOLFOX7 for six cycles followed by maintenance with leucovorin5-fluorouracil alone (maintenance arm), with mFOLFOX7 for six cycles followed by complete cessation of chemotherapy (CFI arm). Median duration of disease control was 13.1 months in patients assigned to the maintenance arm, and 9.2 months in patients assigned to the CFI arm $(P=0.046)$. This study indicated that planned complete discontinuation of chemotherapy had a negative impact on treatment efficacy. ${ }^{15}$ 
Maintenance therapy for mCRC was developed after these two studies involving bevacizumab.

In a phase III study, NO16966, which evaluated the efficacy and safety of bevacizumab added to FOLFOX4 or XELOX as first-line treatment for $\mathrm{mCRC}$, the addition of bevacizumab significantly improved PFS but the effect size was slightly smaller than expected (9.4 months vs 8.0 months, hazard ratio $(\mathrm{HR})=0.83 ; P=0.0023$ ). This was considered to be because the duration of bevacizumab treatment in NO16966 was shorter than in the previous study, and there was a lack of treatment with bevacizumab until PD. ${ }^{3}$ On the other hand, in a randomized phase II study, CONcePT, in which patients were randomized to receive cycles of modified FOLFOX7 plus bevacizumab with eight-cycle blocks of oxaliplatin vs continuous oxaliplatin, when bevacizumab treatment added to oxaliplatin-based chemotherapy was sufficiently continued, it improved PFS (median TTF 5.7 months vs 4.2 months; median PFS 12.0 months vs 7.4 months). ${ }^{20}$ As lessons from NO16966 and CONcePT, the early treatment discontinuation or complete stop for reasons other than disease progression should be avoided as far as possible., ${ }^{3,20}$

Several studies of bevacizumab maintenance therapy have been reported. The CAIRO 3 study demonstrated the superiority of maintenance therapy with capecitabine and bevacizumab toward the observation, after induction therapy with six 3-weekly cycles of XELOX plus bevacizumab. The primary endpoint of median PFS2 was 11.7 months in patients with maintenance therapy, and 8.5 months in patients with observation $(P<0.0001) .{ }^{16}$ In addition, the AIO KRK 0207 study denied the non-inferiority of the observation toward maintenance with fluoropyrimidine-based chemotherapy and bevacizumab, after a 24-week induction therapy with oxaliplatin-based chemotherapy and bevacizumab. The primary endpoint of median time to failure of strategy was 6.1 months in patients with observation, and 6.8 months in patients with maintenance $(P=0.11 ; \mathrm{HR}=1.22[95 \% \mathrm{CI}$, $0.96-1.57]$; non-inferiority margin $\mathrm{HR}=1.466) .{ }^{17}$

In this cohort of 40 patients treated with XELOX plus bevacizumab, 14 patients $(35.0 \%)$ received a certain number of cycles of XELOX plus bevacizumab followed by maintenance with capecitabine plus bevacizumab or capecitabine alone. However, among 20 patients who received eight or more cycles of XELOX plus bevacizumab, eleven patients $(55 \%)$ received maintenance therapy with capecitabine plus bevacizumab or capecitabine alone (Figure 1). Among these eleven patients, seven were treated until PD, and treatment was discontinued in two patients due to radiological CR.
Among the remaining nine patients (45\%) who were not treated with maintenance therapy, three were treated until PD, two discontinued treatment due to radiological CR, and one underwent resection due to response to XELOX plus bevacizumab.

On the other hand, the "planned" strategy of treatment discontinuation or CFI for mCRC cannot be conducted, but CFI or chemotherapy holiday as a result of cure of diseases, such as radiological CR or metastasectomy, plays a crucial role in improving survival of patients with mCRC.

Adam et $\mathrm{al}^{21}$ reported that the 5-year survival rate was $33 \%$ in patients with colorectal liver metastases who were assessed as initially unresectable but reassessed as resectable after response to chemotherapy and underwent secondary hepatic resections, although the 5-year survival rate was $0 \%$ to $5 \%$ in patients with unresectable mCRC. Recently, the CALGB/SWOG 80405 study reported that 132 patients underwent hepatic resections and had no evidence of disease, among 1,137 patients enrolled in the study with unresectable mCRC with wild-type KRAS Exon 2 tumor, but resection of metastases was intended as a result of tumor response in $15 \%$ of these patients. ${ }^{22}$ The median OS of these 132 patients was 64.7 months. Moreover, in both the BOXER study, a phase II study of XELOX plus bevacizumab for liver-limited diseases (LLD) from colorectal cancer, and the CELIM study, a phase II study of FOLFOX/FOLFIRI plus cetuximab for LLD, favorable long-term survival was reported for patients with initially unresectable LLD who responded to chemotherapy and underwent curative secondary resection, although tumor recurrence inevitably occurred. ${ }^{23,24} \mathrm{In}$ the CELIM study, the median OS was 53.9 months, while the median PFS was 15.4 months for 36 patients for whom curative secondary resections were performed. ${ }^{24}$ Thus, a CFI or chemotherapy holiday as a result of tumor response to chemotherapy and hepatectomy contributes to improvement of survival.

In this cohort of 40 patients treated with XELOX plus bevacizumab, among 20 patients who received seven or fewer cycles of XELOX plus bevacizumab, nine patients (45\%) underwent resection as a result of response to XELOX plus bevacizumab. In this cohort study, the median PFS and OS were increased to 13.3 and 38.9 months, respectively, although the median TTF was 5.3 months. This may have resulted from the CFI in these nine patients for whom secondary resection was performed.

This observational cohort study investigated treatment continuity among 40 consecutive patients treated with 
XELOX plus bevacizumab in daily clinical practice. As reported in previous clinical studies, maintenance therapy with capecitabine plus bevacizumab plays an important role in treatment continuity and efficacy, and CFI or chemotherapy holiday resulting from radiological CR or metastasectomy prolonged the PFS and thus improved OS.

\section{Acknowledgments}

The authors thank the following institutions for their cooperation with enrollment and data follow-up: Sano Hospital, Kobe City Medical Center West Hospital, Kobe-Ekisaikai Hospital, Nishikobe Medical Center, and Hyogo Prefectural Nishinomiya Hospital.

The authors also thank Taro Ikumoto from Sano Hospital; Atushi Itami and Saori Goto (now moved to Kyoto University) from Nishikobe Medical Center; and Ryuichi Mikami and Hiroyuki Kayada from Kobe City Medical Center West Hospital especially for their cooperation with data collection. The abstract of this paper was presented at the Gastrointestinal Cancers Symposium, January 16-18, 2014, in San Francisco as a poster presentation with interim findings. The abstract of the poster was published in J Clin Oncol 2014, 32:3s (suppl; abstr 661): http://meeting.ascopubs.org/ cgi/content/short/32/3 suppl/661.

\section{Disclosure}

MK received honoraria from Chugai Pharmaceutical, Yakult Honsha, Merck, and Takeda. TK received honoraria from Chugai Pharmaceutical, Yakult Honsha, Merck, Takeda, Taiho, Bayer, and Eli Lilly. AT received honoraria from Chugai Pharmaceutical, Yakult Honsha, Merck, BristleMyers Squibb, Takeda, and Taiho.

The other authors report no conflicts of interest in this work.

\section{References}

1. Tournigand C, André T, Achille E, et al. FOLFIRI followed by FOLFOX6 or the reverse sequence in advanced colorectal cancer: a randomized GERCOR study. J Clin Oncol. 2004;22(2):229-237.

2. Hurwitz H, Fehrenbacher L, Novotny W, et al. Bevacizumab plus irinotecan, fluorouracil, and leucovorin for metastatic colorectal cancer. N Engl J Med. 2004;350(23):2335-2342.

3. Saltz LB, Clarke S, Díaz-Rubio E, et al. Bevacizumab in combination with oxaliplatin-based chemotherapy as first-line therapy in metastatic colorectal cancer: a randomized phase III study. J Clin Oncol. 2008;26(12): 2013-2019.

4. Van Cutsem E, Köhne CH, Hitre E, et al. Cetuximab and chemotherapy as initial treatment for metastatic colorectal cancer. $N$ Engl J Med. 2009;360(14):1408-1417.

5. Bokemeyer C, Bondarenko I, Makhson A, et al. Fluorouracil, leucovorin, and oxaliplatin with and without cetuximab in the first-line treatment of metastatic colorectal cancer. J Clin Oncol. 2009;27(5):663-671.
6. Douillard JY, Siena S, Cassidy J, et al. Randomized, phase III trial of panitumumab with infusional fluorouracil, leucovorin, and oxaliplatin (FOLFOX4) versus FOLFOX4 alone as first-line treatment in patients with previously untreated metastatic colorectal cancer: the PRIME study. J Clin Oncol. 2010;28(31):4697-4705.

7. Grothey A, Van Cutsem E, Sobrero A, et al. Regorafenib monotherapy for previously treated metastatic colorectal cancer (CORRECT): an international, multicentre, randomised, placebo-controlled, phase 3 trial. Lancet. 2013;381(9863):303-312.

8. Mayer RJ, Van Cutsem E, Falcone A, et al. Randomized trial of TAS102 for refractory metastatic colorectal cancer. $N$ Engl J Med. 2015; 372:1909-1919.

9. Schwartzberg LS, Rivera F, Karthaus M, et al. PEAK: a randomized, multicenter phase II study of panitumumab plus modified fluorouracil, leucovorin, and oxaliplatin (mFOLFOX6) or bevacizumab plus mFOLFOX6 in patients with previously untreated, unresectable, wildtype KRAS exon 2 metastatic colorectal cancer. J Clin Oncol. 2014; 32(21):2240-2247.

10. Heinemann V, von Weikersthal LF, Decker T, et al. FOLFIRI plus cetuximab versus FOLFIRI plus bevacizumab as first-line treatment for patients with metastatic colorectal cancer (FIRE-3): a randomised, open-label, phase 3 trial. Lancet Oncol. 2014;15(10):1065-1075.

11. Venook AP, Niedzwiecki D, Lenz HJ, et al. CALGB/SWOG 80405: phase III trial of irinotecan/5-FU/leucovorin (FOLFIRI) or oxaliplatin/5-FU/leucovorin (mFOLFOX6) with bevacizumab (BV) or cetuximab (CET) for patients (pts) with KRAS wild-type (wt) untreated metastatic adenocarcinoma of the colon or rectum (MCRC). Proc Am Soc Clin Oncol. 2014;32(suppl 5):LBA3 (abstr).

12. Buyse M, Burzykowski T, Carroll K, et al. Progression-free survival is a surrogate for survival in advanced colorectal cancer. J Clin Oncol. 2007;25(33):5218-5224.

13. Tang PA, Bentzen SM, Chen EX, Siu LL. Surrogate end points for median overall survival in metastatic colorectal cancer: literature-based analysis from 39 randomized controlled trials of first-line chemotherapy. J Clin Oncol. 2007;25(29):4562-4568.

14. Tournigand C, Cervantes A, Figer A, et al. OPTIMOX1: a randomized study of FOLFOX4 or FOLFOX7 with oxaliplatin in a stop-and-Go fashion in advanced colorectal cancer - a GERCOR study. J Clin Oncol. 2006;24(3):394-400.

15. Chibaudel B, Maindrault-Goebel F, Lledo G, et al. Can chemotherapy be discontinued in unresectable metastatic colorectal cancer? The GERCOR OPTIMOX2 Study. J Clin Oncol. 2009;27(34):5727-5733.

16. Simkens LH, van Tinteren H, May A, et al. Maintenance treatment with capecitabine and bevacizumab in metastatic colorectal cancer (CAIRO3): a phase 3 randomised controlled trial of the Dutch Colorectal Cancer Group. Lancet. 2015;385(9980):1843-1852.

17. Arnold D, Graeven U, Lerchenmuller CA, et al. Maintenance strategy with fluoropyrimidines (FP) plus Bevacizumab (Bev), Bev alone, or no treatment, following a standard combination of FP, oxaliplatin $(\mathrm{Ox})$, and Bev as first-line treatment for patients with metastatic colorectal cancer (mCRC): a phase III non-inferiority trial (AIO KRK 0207). J Clin Oncol. 2014;32(suppl 5): abstr 3503.

18. Cassidy J, Clarke S, Díaz-Rubio E, et al. Randomized phase III study of capecitabine plus oxaliplatin compared with fluorouracil/folinic acid plus oxaliplatin as first-line therapy for metastatic colorectal cancer. J Clin Oncol. 2008;26(12):2006-2012.

19. Doi T, Boku N, Kato K, et al. Phase I/II study of capecitabine plus oxaliplatin (XELOX) plus bevacizumab as first-line therapy in Japanese patients with metastatic colorectal cancer. Jpn J Clin Oncol. 2010; 40(10):913-920.

20. Hochster HS, Grothey A, Hart L, et al. Improved time to treatment failure with an intermittent oxaliplatin strategy: results of CONcePT. Ann Oncol. 2014;25(6):1172-1178.

21. Adam R, Delvart V, Pascal G, et al. Rescue surgery for unresectable colorectal liver metastases downstaged by chemotherapy: a model to predict long-term survival. Ann Surg. 2004;240(4):644-657. 
22. Venook A, Niedzwiecki D, Lenz H, et al. CALGB/SWOG 80405: analysis of patients undergoing surgery as part of treatment strategy. Ann Oncol. 2014;25(suppl 4): abstr LBA10.

23. Wong R, Cunningham D, Barbachano Y, et al. A multicentre study of capecitabine, oxaliplatin plus bevacizumab as perioperative treatment of patients with poor-risk colorectal liver-only metastases not selected for upfront resection. Ann Oncol. 2011;22(9):2042-2048.
24. Folprecht G, Gruenberger T, Bechstein W, et al. Survival of patients with initially unresectable colorectal liver metastases treated with FOLFOX/cetuximab or FOLFIRI/cetuximab in a multidisciplinary concept (CELIM study). Ann Oncol. 2014;25(5):1018-1025.

\section{Publish your work in this journal}

OncoTargets and Therapy is an international, peer-reviewed, open access journal focusing on the pathological basis of all cancers, potential targets for therapy and treatment protocols employed to improve the management of cancer patients. The journal also focuses on the impact of management programs and new therapeutic agents and protocols on

\section{Dovepress}

\footnotetext{
Submit your manuscript here: http://www.dovepress.com/oncotargets-and-therapy-journal
}

patient perspectives such as quality of life, adherence and satisfaction. The manuscript management system is completely online and includes a very quick and fair peer-review system, which is all easy to use. Visit http://www.dovepress.com/testimonials.php to read real quotes from published authors. 